\title{
Ferromagnetism in One Layer of Self-organized InMnAs Quantum Dots
}

\author{
Im Taek Yoon • Sejoon Lee $\cdot$ Yoon Shon · \\ Seung Woong Lee · Tae Won Kang
}

Received: 20 May 2010 / Accepted: 29 August 2010 / Published online: 18 September 2010

(C) The Author(s) 2010. This article is published with open access at Springerlink.com

\begin{abstract}
One layer of self-assembled InMnAs quantum dots with InGaAs barrier was grown on high-resistivity (100) p-type GaAs substrates by molecular beam epitaxy (MBE). A presence of ferromagnetic structure was confirmed in the InMnAs dilute magnetic quantum dots. The one layer of self-assembled InMnAs quantum dots was found to be semiconducting, and have ferromagnetic ordering with a Curie temperature, $T_{C}=80 \mathrm{~K}$. It is likely that the ferromagnetic exchange coupling of sample with $T_{C}=80 \mathrm{~K}$ is hole-mediated resulting in Mn substituting Ge. PL emission spectra of InMnAs samples grown at temperature of $210^{\circ} \mathrm{C}$ and $285^{\circ} \mathrm{C}$ show that the interband transition peak centered at $1.31 \mathrm{eV}$ comes from the InMnAs quantum dot.
\end{abstract}

Keywords Ferromagnetism · Quantum dots ·

Photoluminescence

\section{Introduction}

Dilute magnetic semiconductors (DMSs), often referred to as semimagnetic semiconductors that are based on III-V semiconductors, have attracted a great deal of attention recently because of their potential application in spintronic devices that exploit the charge and spin of electrons [1-4]. The lattices of these materials consist of magnetic ions partially substituting for some of the cations, thereby inducing

I.T. Yoon $(\bowtie) \cdot$ S. Lee $\cdot$ Y. Shon · T.W. Kang

Quantum Functional Semiconductor Research Center, Dongguk

University, Seoul 100-715, Korea

e-mail: ityoon@dongguk.edu

S.W. Lee

Department of Physics, Konkuk University, Seoul 143-701, Korea a local magnetic moment in the lattice and donating carriers into the system. The ferromagnetic nature of these materials is caused by the exchange interaction between localized magnetic moments introduced by the magnetic ions and the carrier spins. Therefore, for device applications, it is desirable to find materials that exhibit ferromagnetism at as high a temperature as possible. Though the concentration of $\mathrm{Mn}$ in GaMnAs has reached $10 \%$, the Curie temperature is no more than $150 \mathrm{~K}[5,6]$. Thus some researchers have begun to focus on a material such as quantum dots that can provide both excellent optical and magnetic properties. Semiconductor quantum dots (QDs) with peculiar zero-dimensional (0-D) feature of carrier wavefunction have been receiving considerable attention due to scientific importance as well as potential applications in a wide range of devices such as QD-based memory devices [7], single-photon emitters [8], laser diodes [9], and infrared photodetectors [10]. The nonradiative probability of carriers even when there are defects in the materials can be reduced because of the restriction of the carriers in QDs in three dimensions (3D). So it is expected that nanostructures of DMS, such as Mn-including InAs dots, will exhibit interesting magneto-optical properties due to both sp-d exchange interaction effect and strong quantum confinement effect. Furthermore, the confinement effect of a QD structure has been noted to result in a high Curie temperature of the materials. Therefore, InAs dots including Mn atoms are also expected to be used for novel device applications since the structural, electronic, and magnetic properties of InAs dots including Mn atoms have been investigated theoretically and experimentally [11-15].

In this study, we report on the magnetic and optical properties of dilute magnetic InAs dots including Mn atoms grown on (100) GaAs substrates by molecular beam epitaxy (MBE) for novel device applications. Atomic force microscopy (AFM), X-ray diffraction (XRD), superconducting 
quantum interference device (SQUID), photoluminescence (PL) measurements were performed to characterize the samples.

\section{Experimental}

The InMnAs QD heterostructures were fabricated through the Stranski-Krastanov growth technique using an MBE (Riber 2300) system, and the schematic view of the sample cross section is shown in Fig. 1. The prepared (100) GaAs substrates were thermally deoxidized and cleaned at $480^{\circ} \mathrm{C}$ and $525^{\circ} \mathrm{C}$, respectively, in the ultrahigh vacuum MBE chamber $\left(\sim 10^{-10}\right.$ Torr $)$. Onto the substrates, the $0.5 \mu \mathrm{m}$-thick GaAs buffer layer and the $0.1 \mu \mathrm{m}$-thick low temperature (LT)-grown GaAs layer were sequentially grown at $500^{\circ} \mathrm{C}$ and $240-275^{\circ} \mathrm{C}$, respectively. And then, self-organized InMnAs QDs were grown on the LT-grown GaAs buffer layer at $210-285^{\circ} \mathrm{C}$ under high $\mathrm{As}_{4}$ overpressure $\left(P_{\mathrm{As}_{4}} \geq 1.8 \times 10^{-5}\right.$ Torr $)$ with an As-to-In flux ratio of 18. During the growth of InMnAs QDs, the growth rate was sufficiently low as $0.05 \mathrm{ML} / \mathrm{s}$. The reflection high energy electron diffraction (RHEED) pattern was streaky at the initial stage for the growth of QDs, and after growing $2 \mathrm{ML}$ of InMnAs, the RHEED pattern changed to be dim and turned to be spotty after the growth of $3.8 \mathrm{ML}$ of InMnAs with the growth interruption for $30 \mathrm{~s}$. The average length and average height of InMnAs QDs were confirmed to be $\sim 35 \mathrm{~nm}$ and $\sim 12 \mathrm{~nm}$, respectively, by atomic force microscopy (AFM) measurements. The dot density of $\sim 10^{9} \mathrm{~cm}^{-2}$ was also estimated from the AFM surface image. The fraction $(x)$ of $\mathrm{Mn}$ in $\operatorname{In}_{1-x} \mathrm{Mn}_{x}$ As QDs was estimated to be 0.03 through the X-ray diffraction (XRD) measurements using InMnAs bulk layers grown on LT-GaAs/GaAs buffer/(100) GaAs at $285^{\circ} \mathrm{C}$ under the same flux condition. After the growth of InMnAs QDs, the InGaAs capping layer with the thickness of $25 \AA$ was subsequently grown at the same temperature. Oneperiod of InGaAs (25 $\mathrm{A}) / \mathrm{InMnAs}$ QDs were grown, and finally, the $0.1 \mu \mathrm{m}$-thick LT-GaAs as a passivation layer was grown onto the sample. The magnetic properties were investigated using a SQUID, and a high-sensitivity $\left(10^{-8} \mathrm{emu}\right)$ alternating gradient magnetometer (AGM). In all the measurements, the magnetic field was applied parallel to the sample surface. X-ray diffraction (XRD) was used to analyze the structural and morphological properties of the asprepared and annealed samples. PL measurements were carried out at low temperature $(14 \mathrm{~K})$ and room temperature $(300 \mathrm{~K})$ using typical visible-to-near-infrared monochromator system with a closed-cycle He refrigerator $(10 \mathrm{~K})$ and an Ar-ion laser $(\lambda=514.5 \mathrm{~nm})$, and the luminescence signals were detected by a photomultiplier tube.

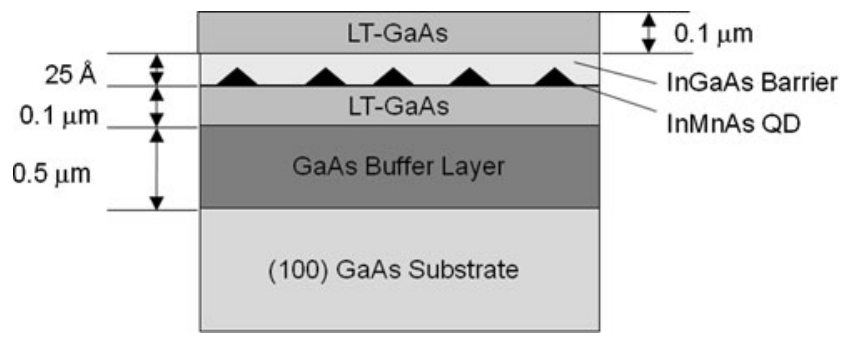

Fig. 1 Schematic view of the one-period InMnAs QD heterostructure sample grown by MBE

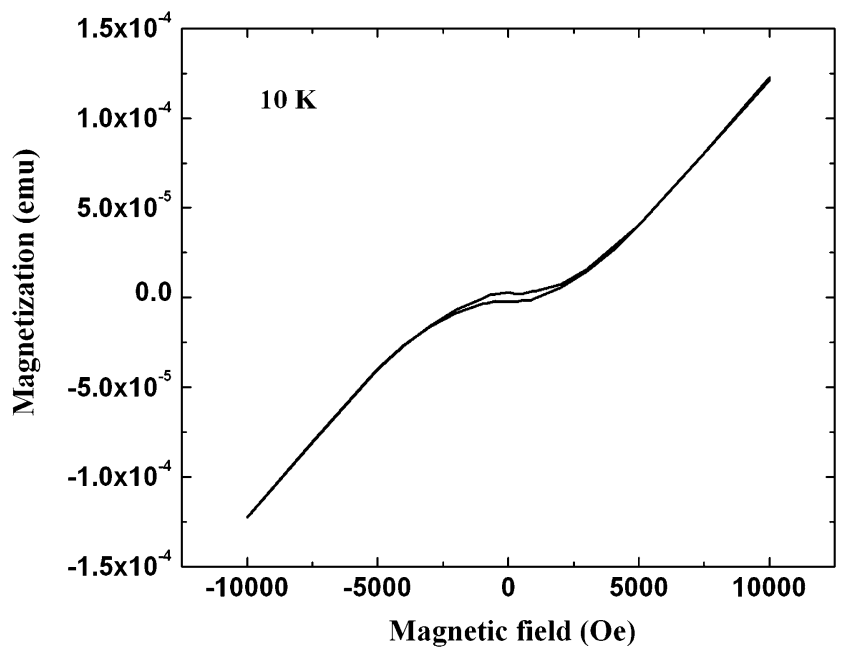

Fig. 2 A ferromagnetic hysteresis loop of one-stacked InMnAs sample grown at $210^{\circ} \mathrm{C}$ obtained by SQUID magnetometry at $10 \mathrm{~K}$

\section{Results and discussion}

Figure 1 shows the schematic view of the one-period InMnAs QD heterostructure sample grown by MBE. Figure 2 shows the magnetic-field dependent magnetization, $\mathrm{M}$, for the one-stacked InMnAs sample grown at $210^{\circ} \mathrm{C}$ at 10 $\mathrm{K}$, with the magnetic field applied parallel to the plane of the film using an AGM. Clearly elongated hysteresis loops are observed in Fig. 2, indicating that a ferromagnetic structure is present. The diamagnetic background of the substrate was subtracted from the data. The temperature dependence of the magnetization curve, $M(T)$, for the one-period InMnAs QD heterostructure sample grown by MBE is presented in Fig. 3. A magnetic field of 500 Oe was applied parallel to the film plane in the $M-T$ measurement. It can be seen that the Curie temperature for the sample is $80 \mathrm{~K}$. For convenience, the temperature at which the magnetic moment vanishes under a small magnetic field is taken as the Curie temperature $T_{C}$, which is seen in Fig. 3. Therefore, our data excludes the existence of other ferromagnetic compounds that have the Curie temperature higher than $400 \mathrm{~K}$. It is well known that both GaMn and MnAs clusters present room temperature ferromagnetism [16]. A Curie temperature $T_{C}$ of $350 \mathrm{~K}$ is 


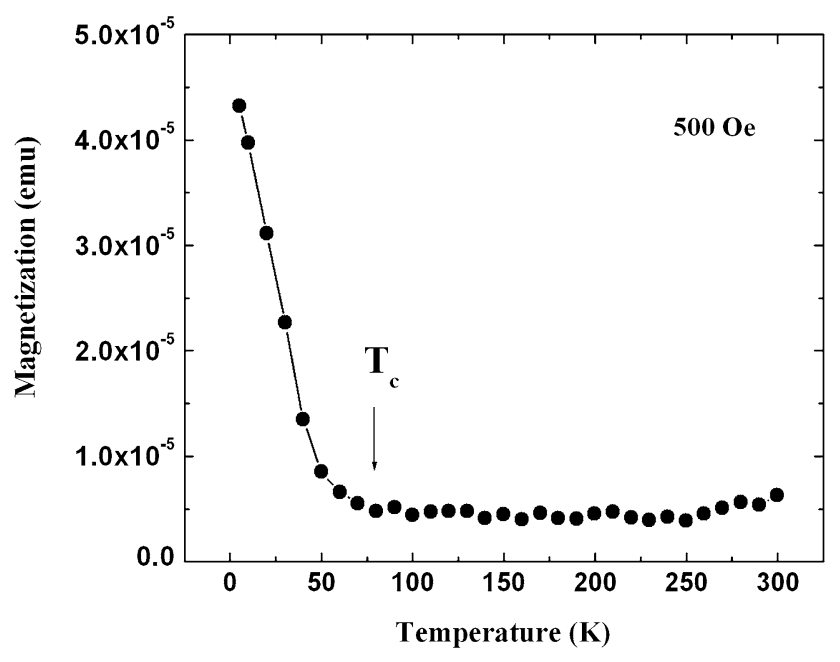

Fig. 3 Temperature dependence of magnetization of one-stacked InMnAs sample grown at $210^{\circ} \mathrm{C}$ obtained by SQUID magnetometry with a magnetic field of 500 Oe. The arrow indicates a Curie temperature of $80 \mathrm{~K}$

reported in InMnAs self-organized diluted magnetic quantum dot (DMQD) samples grown by low-temperature solidsource molecular-beam epitaxy [17]. The authors proposed that the reason for a high Curie temperature is due to the effects of magnetic and structural disorder introduced by a random incorporation and inhomogeneous distribution of Mn atoms amongst the InAs quantum dots.

The observed temperature-dependent magnetization curve $M(T)$ appears to be very different from the usual convex $M(T)$ curve expected from the Weiss mean-field theory $[18,19]$. The temperature-dependent magnetization shows an unusual outwardly concave shape that is consistent with behavior of GeMn system [20], suggesting a signature of charge carrier localization. It has been shown that such a non-mean-field like behavior in $M(T)$ curve is much more typical characteristic for a DMS based on the model of bound magnetic polarons [21, 22]. No itinerant hole-based picture is possible, and therefore the RKKY-type scenario discussed extensively in the framework of GaMnAs magnetization $[1,2]$ simply does not apply to our samples. The bound magnetic polaron (BMP) percolation picture [21] as discussed in the framework of strongly semi-insulating DMS materials may thus be related. Chen et al. [23] suggested that the ferromagnetism of MnGe nanostructure is hole-mediated and the spin of localized carriers can polarize the surrounding magnetic impurities, leading to a socalled bound magnetic polaron [24] which can be regarded as a DMQD. Therefore, it is likely that the ferromagnetism observed in the sample A with $T_{C}=80 \mathrm{~K}$ is associated with the carriers produced by Mn atoms occupying In sites $\left(\mathrm{Mn}_{\text {In }}\right)$. This behavior further suggests that the ferromagnetic exchange coupling is hole-mediated and is consistent with other DMS systems $[1,20]$.

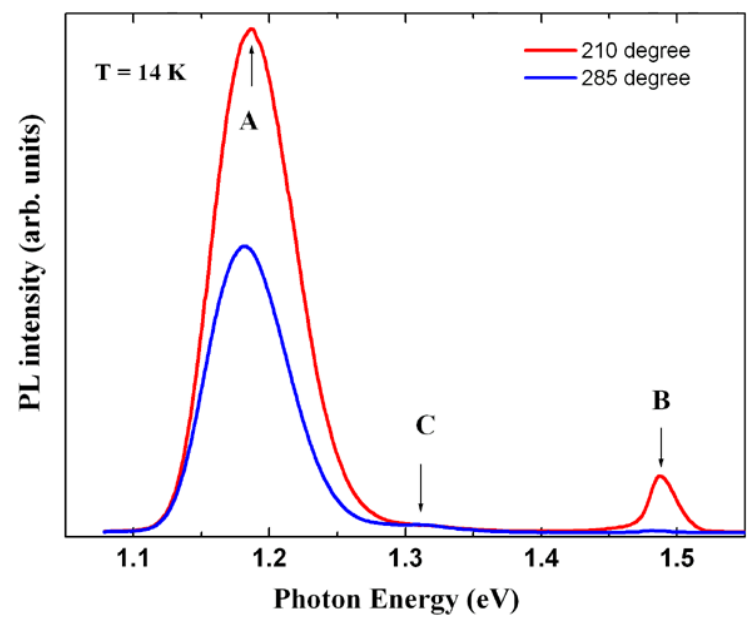

Fig. 4 PL spectra of one-stacked InMnAs samples grown at $210^{\circ} \mathrm{C}$ and $285^{\circ} \mathrm{C}$ measured under same excitation conditions at $14 \mathrm{~K}$

Figure 4 shows the PL emission spectra of InMnAs samples grown at temperature of $210^{\circ} \mathrm{C}$ and $285^{\circ} \mathrm{C}$ measured under same excitation conditions at $14 \mathrm{~K}$. As shown in Fig. 4, two peaks (A and B) from InMnAs dot were observed. The peak A centered at $1.19 \mathrm{eV}$ comes from the InAs quantum dots without $\mathrm{Mn}$ [15]. A reduction in the intensity of the peak A occurs with increasing growth temperatures. The reason why this reduction in the intensity of emission occurs with increasing growth temperature is due to the increased thermal energy of carriers. The peak B centered at $1.49 \mathrm{eV}$ comes from the InAs wetting layer which is the reported value for the two-dimensional InAs wetting layer of $1.4-1.5 \mathrm{eV}$ [25]. The peak B is completely quenched out in the InMnAs dot grown at $285^{\circ} \mathrm{C}$. The peak $\mathrm{C}$ centered at $1.31 \mathrm{eV}$ coming from the InMnAs quantum dot is due to the band-to-band transition from the ground electronic subband to the ground heavy-hole sub-band (E1-HH1) and almost weakly observed. This peak centered at $1.31 \mathrm{eV}$, identified as the interband transition, was observed in the disklike Mn-including InAs dots grown by metaloganic molecular beam epitaxy [12] and InAs quantum dots fabricated $\mathrm{Mn}$ implantation [15]. The PL peak centered at $1.31 \mathrm{eV}$ for the 2 monolayers of InAs sandwiched with GaAs layers was also reported [26]. Further work on the magneto-optical experiments on InMnAs nanostructure is in progress.

\section{Conclusions}

In summary, one layer of self-assembled InMnAs quantum dots with InGaAs barrier was grown on high-resistivity (100) p-type GaAs substrates by MBE. It can be concluded that the ferromagnetic exchange coupling of InMnAs quantum dots with a Curie temperature of $80 \mathrm{~K}$ is hole-mediated, resulting in Mn substituting Ge. PL emission spectra of InMnAs samples grown at $210^{\circ} \mathrm{C}$ and $285^{\circ} \mathrm{C}$ show that the 
inter-band transition peak centered at $1.31 \mathrm{eV}$ comes from the InMnAs quantum dot.

Acknowledgements This research was supported by Basic Science Research Program through the National Research Foundation of Korea (NRF) funded by the Ministry of Education, Science and Technology (2010-0021905) and QSRC of Dongguk University.

Open Access This article is distributed under the terms of the Creative Commons Attribution Noncommercial License which permits any noncommercial use, distribution, and reproduction in any medium, provided the original author(s) and source are credited.

\section{References}

1. Ohno, H.: Science 281, 951 (1998)

2. Munekata, H., Ohno, H., von Molnar, S., Segmuller, A., Chang, L.L., Esaki, L.: Phys. Rev. Lett. 63, 1849 (1989)

3. Ohno, H., Shen, A., Matsukura, F., Oiwa, A., Endo, A., Katsumoto, S., Iye, Y.: Appl. Phys. Lett. 69, 363 (1996)

4. Munekata, H., Zaslavsky, A., Fumagalli, P., Ganbino, R.J.: Appl. Phys. Lett. 63, 2929 (1993)

5. Edmonds, W., Wang, K.Y., Campion, R.P., Neumann, A.C., Foxon, C.T., Gallagher, B.L., Main, P.C.: Appl. Phys. Lett. 81, 3010 (2002)

6. Edmonds, K.W., Wang, K.Y., Campion, R.P., Neumann, A.C., Farley, N.R.S., Gallagher, B.L., Foxon, C.T.: Appl. Phys. Lett. 81, 4991 (2002)

7. Petroff, P.M., Lorke, A., Imanoglu, A.: Phys. Today 54, 46 (2001)

8. Yuan, Z., Kardynal, B.E., Stevenson, R.M., Shields, A.J., Lobo, C.J., Cooper, K., Beattie, N.S., Ritchie, D.A., Pepper, M.: Science 295, 102 (2002)

9. Kuntz, M., Fiol, G., Laemmlin, M., Bimberg, D., Thompson, M.G., Tan, K.T., Marinelli, C., Penty, R.V., White, I.H., Ustinov, V.M., Zhukov, A.E., Shernyakov, Yu.M., Kovsh, A.R.: Appl. Phys. Lett. 85, 843 (2004)
10. Jiang, J., Mi, K., Tsao, S., Zhang, W., Lim, H., O’Sullivan, T., Sills, T., Razeghi, M., Brown, G.J., Tidrow, M.Z.: Appl. Phys. Lett. 84, 2232 (2004)

11. Holub, M., Chakrabarti, S., Fathpour, S., Bhattacharya, P., Lei, Y., Ghosh, S.: Appl. Phys. Lett. 85, 973 (2004)

12. Zhou, Y.K., Asahi, H., Asakura, J., Okumura, S., Asami, K., Gonda, S.: J. Cryst. Growth 221, 605 (2000)

13. Primus, J.-L., Choi, K.-H., Trampert, A., Yakunin, A., Ferre, J., Wolter, J., Van Roy, W., De Boeck, J.: J. Cryst. Growth 280, 32 (2005)

14. Zhou, Y.K., Asahi, H., Asakura, J., Okumura, S., Asami, K., Gonda, S.: J. Cryst. Growth 221, 605 (2000)

15. Hu, L.J., Chen, Y.H., Ye, X.L., Jiao, Y.H., Shi, L.W., Wang, Z.G.: Physica E 40, 2869 (2008)

16. Katscher, H., et al.: In: Gmelin Handbook of Inorganic and Organometallic Chemistry, vol. 56. Springer, Berlin (1983)

17. Holub, M., Chakrabarti, S., Fathpour, S., Bhattacharya, P., Lei, Y., Ghosh, S.: Appl. Phys. Lett. 85, 973 (2004)

18. Ashcroft, M.W., Mermin, N.D.: Solid State Physics. Saunders College, Philadelphia (1976)

19. Kittel, C.: Introduction to Solid State Physics. Wiley, New York (1996)

20. Park, Y.D., Hanbicki, A.T., Erwin, S.C., Hellberg, C.S., Sullivan, J.M., Mattson, J.E., Ambrose, T.F., Wilson, A., Spanos, G., Jonker, B.T.: Science 295, 651 (2002)

21. Sarma, S.D., Hwang, E.H., Kaminski, A.: Phys. Rev. B 67, 155201 (2003)

22. Bihler, C., Jaeger, C., Vallaitis, T., Gjukic, M., Brandt, M.S., Pippel, E., Woltersdorf, J., Gösele, U.: Appl. Phys. Lett. 88, 112506 (2006)

23. Chen, J., Wang, K.L., Galatsis, K.: Appl. Phys. Lett. 90, 012501 (2007)

24. Morresi, L., Pinto, N., Ficcadenti, M., Murri, R., D’Orazio, F., Lucari, F.: Mater. Sci. Eng. B 126, 197 (2006)

25. Phillips, J., Kamath, K., Bhattacharya, P.: Appl. Phys. Lett. 72, 2020 (1998)

26. Xu, Z.Y., Lu, Z.D., Yang, X.P., Yuan, Z.L., Zheng, B.Z., Xu, J.Z.: Phys. Rev. B 54, 11528 (1996) 